\title{
Transformation of the Transaction Cost and the Agency Cost in an Organization and the Applicability of Blockchain-A Case Study of Peer-to-Peer Insurance
}

OPEN ACCESS

Edited by:

Horst Treib/maier,

MODUL University Vienna, Austria

Reviewed by:

James Burnie,

Eversheds Sutherland (International)

LLP, United Kingdom

Janine Hacker,

University of Zurich, Switzerland

Ingrid Bauer,

University of Zurich, Switzerland in collaboration with reviewer $\mathrm{JH}$

*Correspondence: Ruo-Ting Sun rsun12@illinois.edu

Specialty section: This article was submitted to Non-Financial Blockchain, a section of the journal

Frontiers in Blockchain

Received: 29 August 2019 Accepted: 27 April 2020

Published: 21 May 2020

Citation:

Sun R-T, Garimella A, Han W, Chang H-L and Shaw MJ (2020)

Transformation of the Transaction

Cost and the Agency Cost in an Organization and the Applicability of Blockchain-A Case Study of Peer-to-Peer Insurance.

Front. Blockchain 3:24.

doi: 10.3389/fbloc.2020.00024

\author{
Ruo-Ting Sun ${ }^{1 *}$, Aravinda Garimella ${ }^{1}$, Wencui Han ${ }^{1}$, Hsin-Lu Chang ${ }^{2}$ and Michael J. Shaw ${ }^{1}$ \\ ${ }^{1}$ Department of Business Administration, University of Illinois Urbana-Champaign, Champaign, IL, United States, \\ ${ }^{2}$ Department of Management Information System, National Chengchi University, Taipei, Taiwan
}

Blockchain supports a variety of decentralized applications enabled by its immutable, decentralized, and trustless properties. However, there are no unifying criteria for blockchain architecture across the organizations and business models. This variance has created complex and diverse blockchain products. Costs in every economic exchange with partners are associated with two metrics: transaction costs due to market imperfections and agency costs due to conflict of interest and information asymmetry in an organization. To understand the effectiveness of economic activities by blockchain intervention and facilitate strategic alignment, we use transaction cost and agency cost as theoretical lenses to explore the impacts of blockchain, discuss the transformation of those costs, and support our arguments using a case study. Our study proposes that blockchain technology brings two more benefits, trust and transparency, to the existing Internet-based business services, and helps improve corporate governance. Smart contracts improve the execution time of transactions significantly and increase transaction volume rapidly. As the internet shifts hierarchies toward electronic markets, lack of trust between peers inhibits exchanges. Blockchain applications provide a framework for building trust between peers through its consent mechanism, which allows organizations to construct trust and operate in a more decentralized manner. Thus, by including blockchain in the current Internet infrastructure, the decision boundary of organization forms would extend outward. Finally, the transformation of costs in different stages of the blockchain transition, as described in our study, has important managerial implications for the organization structure and the role of third parties. Blockchain does not assume away transaction and agency costs but pushes the transformation of the two, forming a more efficient economic entity. This study contributes to the academia and the industry. We first add to the understanding of blockchain from the perspective of exchange technology. Second, we contribute to the prediction of organization boundaries. Third, the shift in the role of third parties supports the transaction cost theory in terms of controlling opportunism. Lastly, this study facilitates the development of blockchain business models and contributes to the practice.

Keywords: blockchain, transaction cost, agency cost, exchange technology, organization costs evolution, blockchain applicability, peer-to-peer insurance 


\section{INTRODUCTION}

Blockchain is an emerging information technology (IT) that supports a variety of decentralized applications enabled by its immutable, decentralized, and trustless properties. Despite its promising future, there are concerns that blockchain applications are overpromised and underdelivered. Some researchers argue that the same tasks addressed by blockchain can also be done by alternative technologies more efficiently ${ }^{1}$. The necessity and the impact of blockchain technology vary across organizations and business models. However, with the hype of block chain technology, corporates are pouring funds into blockchain technology, developing pilots, and proofs-of-concept, but little progress has been made. IT sectors are devoted to optimizing the performance of the system, and most existing studies discuss the potential of blockchain use cases from the perspective of General-Purpose Technology (GPT) (Davidson et al., 2018). GPT focuses on the improvement of multifactor productivity of the system. However, blockchain applications have no unifying criteria for blockchain architecture across organizations and business models. This variance has created complex and diverse blockchain products, so-called blockchain hype, while the applicability of blockchain is underexplored. While focusing on the functionality of a blockchain system, we may narrow the scope to Information System (IS) constructs and overlook organizational-wise variables (Agarwal and Lucas, 2005).

Managers should think outside the chaotic phenomena of blockchain hype and understand how new technology can integrate with existing business processes. A firm-level perspective to re-examine blockchain can be provided through Exchange Technology (ET), which emphasizes the efficiency and the scope of transactions in market exchanges between economic entities (Davidson et al., 2018). Costs in every economic exchange with partners are associated with two metrics: transaction costs due to market imperfections and agency costs due to conflict of interest and information asymmetry in an organization. To understand the effectiveness of economic activities by blockchain intervention and facilitate strategic alignment, we use transaction cost and agency cost as theoretical lenses to explore the impacts of blockchain, discuss the transformation of those costs, and support our arguments using a case study. This study aims to provide managerial insights to examine the applicability of blockchain with respect to the existing business processes of an organization. We seek to answer the following research questions.

Research question 1: What effects does the introduction of blockchain technology have on the boundaries of an organization with respect to IT infrastructure? We develop proposition 1 in section The Impact of Blockchain on Transaction Cost and provide our analysis in section A TwoStage Cost Transformation in Blockchain Transition.

Research question 2: How does the introduction of blockchain transform the transaction costs of a firm? Propositions 2a, 2b, and $2 \mathrm{c}$ are developed for each type of transaction costs in section The Impact of Blockchain

${ }_{1}^{1}$ Blockchain's Occam problem (https://www.mckinsey.com/industries/financialservices/our-insights/blockchains-occam-problem). on Transaction Cost and supported by our case discussion in section Transformation of the Transaction Costs by Blockchain Intervention.

Research question 3: How does the introduction of blockchain transform the agency costs of a firm? Propositions $3 a, 3 b$, and $3 c$ are constructed based on each type of agency costs in section The Impact of Blockchain on Agency Cost and supported by our case discussion in section Transformation of the Agency Costs by Blockchain Intervention.

Research question 4: How do the new transaction costs and agency costs interact and what effects does this have on the organization when adopting blockchain technology to the existing business process? We introduce the last proposition (Proposition 4) in section The Impact of Blockchain on Agency Cost and demonstrate our analysis in section Transformation of the Transaction Costs by Blockchain Intervention and section First-stage Transformation.

We use transaction cost theory and agency theory to examine the interaction between the determinants of the two theories and blockchain properties. We then study how the interaction effects impact the corresponding cost variables in the two theories. The discussed cost transformations are laid out as our research propositions. A blockchain company, InsurePal, is selected to explore our discussed cost transformations. Through the company's official documents, we select a business process and define the cost variables of the two theories in the scenario to examine how those variables transform when blockchain technology involves. Since blockchain is still evolving, we position our case in different blockchain transition stages and discuss research questions supported by our case scenarios. The rest of paper is organized as follows. In section Background and Related Work, we introduce background information on blockchain and summarize our literature review on the transaction and agency theories. We share our research propositions in section Research Proposition and support these propositions with a case study in section Research Methodology. Finally, in sections Discussion, Conclusion and Limitations, and References, we provide a discussion, highlight our contributions and provide concluding remarks.

\section{BACKGROUND AND RELATED WORK}

\section{Properties of Blockchain}

Blockchain has four main properties: transparency, immutability, programmability, and decentralization. First, a blockchain is a distributed ledger without control from central institutions. Every participant in the network has an identical copy of the ledger, resulting in a transparent shared data source. Second, the transaction data is encrypted and grouped into blocks that are chained together, providing an immutable transaction trajectory, hence the name blockchain (Bahga and Madisetti, 2016; Peters and Panayi, 2016; Cachin and Vukolić, 2017; Zheng et al., 2017; Abadi and Brunnermeier, 2018; Davidson et al., 2018). Third, blockchain is powered by a "smart contract" - a set of self-executing agreements between trading partners. With the programmability of a smart contract, it enables registered peers to validate transactions automatically and anonymously (Peters 
and Panayi, 2016) and decides whether a new block can be appended to the chain in chronological order (Baliga, 2017; Cachin and Vukolić, 2017). A blockchain can be viewed as a permanent database that keeps track of all transaction records (Davidson et al., 2018). Lastly, the credentials data is exposed to all participants, potentially including those with less ethical motives. Bad actors are impeded by the consensus mechanism-a fault-tolerant mechanism used in a blockchain network to ensure security by making data on the blockchain immutable (Baliga, 2017; Cachin and Vukolić, 2017). In the event of a malicious attack, a blockchain system can continue working to a satisfactory level within this decentralized setup (no single point failure) (Bahga and Madisetti, 2016).

\section{Types of Blockchain}

A participant's ability to freely enter a blockchain network determines the level of decentralization in blockchain applications (Abadi and Brunnermeier, 2018). There are three types of blockchain ledger systems-private, permissioned, and permissionless (Zheng et al., 2017). A private blockchain can be deployed by a centralized institution whose clients are not involved in the operation process. A permissioned blockchain is formed by a consortium that can regulate the system. A permissionless blockchain enables users to freely enter the market when they generate enough computation power to interact with the blockchain.

Whether a blockchain adapts to an existing business process depends on its configuration and business context. For example, Peters and Panayi elaborate on the difference of configurations between permissioned and permissionless blockchains (Peters and Panayi, 2016). A permissionless blockchain requires the "anonymous" participant to contribute their computational power to enable a block validation and keep the network alive. Since those participants can freely join the network, the design of incentives is crucial to motivate their participation. The wellknown examples of cryptocurrencies (Bitcoin, Ethereum, and more) are a result of permissionless blockchain.

A permissioned blockchain is designed as a transit product to enable the shift of existing business applications from a private centralized blockchain to a decentralized, permissionless blockchain. The pre-selected and "known" participants cooperate as a consortium to verify transactions. When the members of the consortium are large firms, there is a tendency to operate like "centralized authorities" (Zheng et al., 2017). Thus, alternative consensus mechanisms are required. These two contexts of blockchain ledger systems imply different cost structures of blockchain implementation. This study discusses the transformation of costs during the blockchain evolutionary process.

\section{Transaction Cost Theory}

Transaction costs are developed and classified into three types (Mahoney, 2004). First, search and information costs are incurred to reduce uncertainty before a transaction is executed. Second, bargaining costs are incurred during negotiations before reaching a common agreement. Third, policy and enforcement costs are incurred during the supervision of a contract.
The core concept of transaction cost theory aims to enhance economic efficiency within the process of product or service exchange through the market. In addition to the production costs of goods, transaction costs play a crucial role in finding an efficient economic entity and its decision boundary. Other than the above-mentioned categories of transaction costs, Williamson proposed three determinants of transaction costs-frequency, asset specificity and uncertainty as key dimensions that capture the characteristics of economic exchange between institutions (Williamson, 1975).

Especially, the asset specificity has four types-Site, Physical, Human (Williamson, 1983) and Time (Malone et al., 1987). An asset is site specificity when a natural resource only available at certain location. An asset is physical specificity when a specialized tool is developed for a unique business purpose. An asset is human-specific when the required knowledge or skills are built through a learning-by-doing model with trading partners. An asset is time-specific when the value exchange between the user is dependent on a limited period of time.

Since transaction costs are durable, extant studies have demonstrated the transformation of these costs when a new technology emerges (e.g., telegraph, Internet) and predicted the possible organization forms (Malone et al., 1987; Lajili and Mahoney, 2006). In this study, we further explore where blockchain has surpassed previous interventions provided by the internet.

\section{Agency Cost Theory}

Within an organization, the principal-agent problem stems from conflicts of interests between managers (the agent) and shareholders (the principal) and information asymmetry. Blockchain is predicted to mitigate the principle-agent problem. The principal and agent desire for their counterparts to optimize their interests. A contract is fulfilled to supervise and restrict the agent's behavior. The contract creates monitoring costs, bonding costs, and residual losses (Mahoney, 2004).

The principal can reduce information asymmetry and maintain better control of the parties' interests through regular meetings/reports-monitoring cost. The agent is also required to commit to contractual obligations so that managerial behavior is not harmful to the principal-bonding cost. Sometimes the interests between principals and agents are still misaligned due to uncertainties, contributing to the third agency costs-residual losses. Residual losses are opportunity costs that occur when parties fail to fulfill a contract, despite that contract being optimally established to ensure the interests of both parties.

Two determinants are crucial to mitigate the agent-principal problem: incentive and accountability. First, in the principalagent relationship, it is common for the principal to provide incentives to motivate engagement with their agent and mitigate the moral hazard issue. Empirical evidence extended from Jensen and Meckling's base model (Jensen and Meckling, 1976) concluded that agency cost is inversely related to the agent's economic rewards (Ang et al., 2000). Extant studies also analyzed how incentives contracts impact vertical relationships from the perspective of agency theory (Holmstrom, 1979; Zenger, 1994; Foss, 2003). 
Second, Khan claims that clear accountability of the individuals can reduce the misalignment of interests between the principal and the agent (Khan, 2011). A relationship-specific Internet technology is predicted to reduce the agency costs when the accountability of a task is ambiguous (Jensen and Meckling, 1976). Literature has discussed the two variables in the agency theory to help clarify accountability, such as the measurement of input by task programmability (Ouchi, 1979) and the evaluation of outputs by non-separability (Alchian and Demsetz, 1972). Task programmability describes how the specifications of a task can be formulated in advance. If task programmability is ambiguous, the monitoring effort would be a challenge since a manager is not able to identify and clarify the original input. Non-separability measures the traceability of a task to a specific individual. A manager must take the responsibility to monitor the original outputs when incentives are insufficient or inefficiently allocated.

Blockchain is predicted to mitigate the principle-agent problem. Several studies discuss the potential of blockchain to reduce auditing effort, facilitate decision-making, and improve the involvement of shareholders (Chedrawi and Howayeck, 2018; Lafarre and Van der Elst, 2018; Kaal, 2019). However, few cases and tools are available to evaluate the efficiency of blockchain implementation, and its impact on decision support (Swan, 2015; Yli-Huumo et al., 2016).

\section{RESEARCH PROPOSITION}

\section{The Impact of Blockchain on Transaction Cost}

Blockchain's core values-consensus mechanism and smart contract, may fundamentally change the two types of asset specificity in transaction costs: time specificity (Malone et al., 1987) and human specificity (Williamson, 1983). Since blockchain would not impact the way of obtaining natural resource and the demand of specialized machine for production, we would not discuss site specificity and physical specificity in this study. We propose our propositions based on the interplay of four determinants (frequency, time specificity, human specificity, and uncertainty) in transaction theory, and four blockchain properties (programmability, decentralization, transparency, and immutability).

\section{Time Specificity and Programmability}

To save costs, high-frequency transactions between specific trading partners will increase the likelihood of vertical integration (Williamson, 1985). Time specificity has already undergone shifts since the emergence of the internet. Communications can now be realized through electronic integration by relationship-IT systems rather than physical ownership of the upstream facilities (Lajili and Mahoney, 2006). This has shifted the structure of institutions from hierarchies toward markets, as distinguished by Malone et al. (1987).

\section{Frequency and Decentralization}

Through smart contracts, blockchain can further reduce the execution of time of a transaction, increasing the transaction volume. In a fully decentralized market, participants can freely enter the network to transact with each other via the consensus mechanism (Zheng et al., 2017; Abadi and Brunnermeier, 2018). An institution is far less likely to vertical integrate the trading partners.

We extend the argument by Malone et al. (1987) based on the interaction of the two determinants in transaction theory and the two blockchain properties. Blockchain's programmability saves the transaction time and boosts the frequency of a transaction. The backbone consensus allows the participants to execute the growing number of transactions in a decentralized manner. We propose

Proposition 1. Blockchain will push further the structure of an organization from hierarchies toward electronic markets.

\section{Time Specificity and Transparency}

The transparency and traceability of the blockchain digital ledger will significantly reduce the cost of validating trading partners (Catalini and Gans, 2016). Moreover, the programmability of blockchain facilitates the exchange of digital information (Peters and Panayi, 2016). As a result, the time used for collecting required information on a transaction will also be reduced. We propose

Proposition 2a. The search and information cost will decrease in an organization by adopting blockchain technology to the existing business process.

\section{Human Specificity and Decentralization}

The assumption of limited rationality used in transaction cost economics addresses that opportunistic actions may occur due to information asymmetry, leading to the increase of behavior uncertainty (Williamson, 1985). A traditional intermediary is employed and creates costs for the services of providing credibility and reducing the uncertainty of trading with unknown partners (i.e., bank service). Several studies had provided evidence that the internet facilitates the information exchange and resulting in the removal of intermediaries (Chircu and Kauffman, 1999; Ritchie and Brindley, 2000). Information shared through blockchain is verified through the consensus mechanism, which facilitates agreements from participants on-chain. Trust is constructed between trading partners directly instead of through an intermediary. Blockchain applications ensure that an institution no longer relies on a centralized authority to finalize a transaction. Thus, the traditional intermediaries either disappear or play a new role in the blockchain network (Catalini and Gans, 2016). Such a transformation directly impacts another aspect of asset specificity-human capital. Those human-specific resources are transferred to communicate with more partners on-chain rather than merely dealing with traditional centralized authorities.

\section{Uncertainty and Immutability}

As discussed earlier, the reduction of execution time boosts transaction volume. Each transaction is associated with uncertainty. More negotiations are required to reduce uncertainty and fulfill the needs of each participant and corresponding agreement (Mahoney, 2004). In addition, the append-only mechanism of data structure would lead to high 
cost of maintenance when "errors" occur in the common ledger (Catalini and Gans, 2016). Before reaching a final contract, participants have to pre-define their rights and interests in advance.

The interplay between human specificity and decentralization property shifts the nature of communication structure. The immutability of blockchain mitigates the possibility of an inaccurate data registry by reducing the uncertainty of each pretransaction. The two interaction effects increase the negotiation efforts. Thus, we propose

Proposition $2 \boldsymbol{b}$. The bargaining cost will increase in an organization by adopting blockchain technology to the existing business process.

\section{Uncertainty and Programmability}

The agreements are automatically checked by smart contracts, which also reduces the uncertainty of contract enforcement (i.e., human error) (Catalini and Gans, 2016). Hence, smart contract switches the cost of monitoring compliance of contracts toward more bargaining costs. We then propose the following proposition.

Proposition 2c. The policing and enforcement cost will decrease in an organization by adopting blockchain technology to the existing business process.

Although the initial costs in developing the system and negotiation are higher than non-blockchain systems, the payoff would be greater over time after implementation due to the periodical monitoring by the labor force becoming unnecessary as a result of implementing smart contracts. Such a shift caused by blockchain not only achieves a transparent and auditable common ledger to define "truth," it also enforces the responsibilities of each party through cryptographic and smart contracts, leading to a further reduction in transaction costs (Catalini and Gans, 2016). Thus, by way of controlling opportunism (minimize transaction cost), institutions can be economically effective in interacting with economic partners. Figure 1 illustrates the shift of transaction costs in an organization by adopting a blockchain-based system.

\section{The Impact of Blockchain on Agency Cost}

Agency costs arise from the separation of ownership and control of a company. Moral hazards and conflicts of interest between the agent and principal will always exist so long as there are human factors involved the operation process of an organization (Levinthal, 1988). The introduction of the "smart contract" also changes the nature of agency costs since the contractual obligations can be carried out automatically - a new way to track accountability and distribute incentives.

\section{Incentives and Incentive Protocol}

Appropriate incentives motivate the agent to align with the principal's interest (Jensen and Meckling, 1976). Since blockchain types diverse, different incentive protocols are designed to motivate participants' diligent work (Baliga, 2017; Cachin and Vukolić, 2017). The agent's operation performance is recorded transparently and incentivized accordingly, helping reduce the misalignment of interests. Therefore, the mechanism for determining accountability is compulsory and crucial in managing incentives.

\section{BEFORE BLOCKCHAIN ADOPTION}

\section{AFTER BLOCKCHAIN ADOPTION}
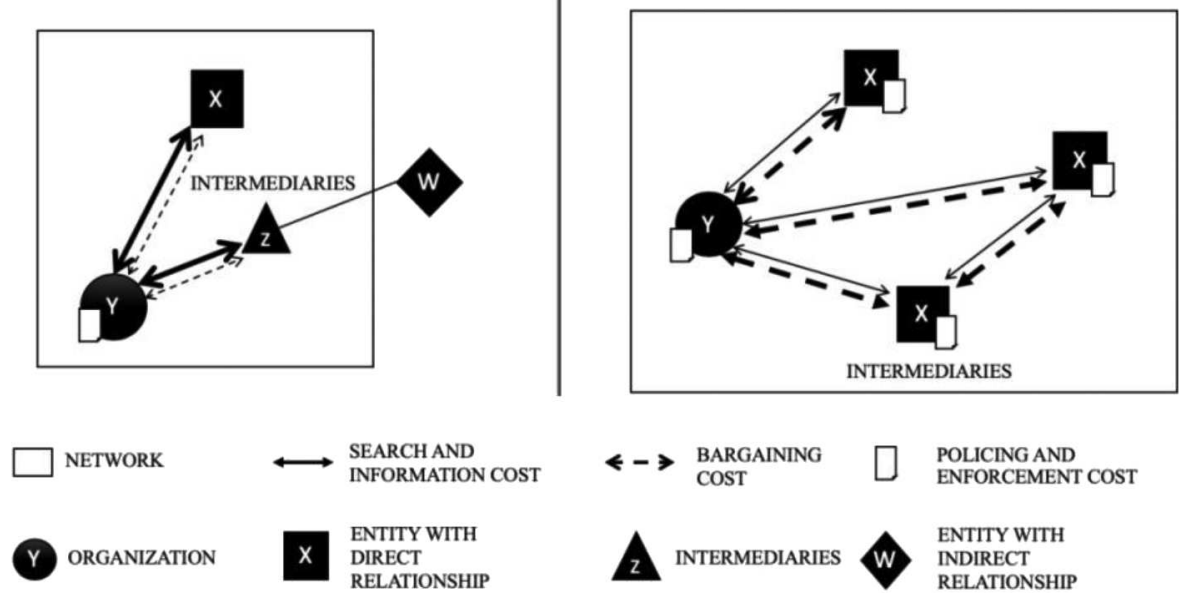

FIGURE 1 | The transformation of transaction costs in the adoption of blockchain technology. The thicknesses of the line represent the amount of costs. Before the adoption of blockchain, organization $Y$ had a direct transaction with $X$ and indirect transactions with $W$ through the intermediary, entity $Z$ and supervised them by a contract. The traditional intermediary $Z$ is either removed or transformed into a new role after adopting blockchain. The nodes are connected, lowering the search and information costs due to the shared ledger. Since the same smart contracts are also owned by each entity, the enforcement of rules is also automatically checked. Therefore, the policing and enforcement costs can be greatly reduced; however, the negotiation efforts would dramatically increase since the pre-defined rules are required for fulfilling each participant's expectations. 


\section{Accountability and Traceability}

Mahoney constructed a framework to determine the choice of a contractual arrangement (determining the obligation of individuals given inputs and outputs) by combining task programmability and non-separability (agency theory) with demand uncertainty, technological uncertainty, and asset specificity (transaction theory) (Mahoney, 1992). Kettinger et al. (1994) also investigated the sustainability of competitive advantage by information technologies that enhance the ability to measure individual inputs and track managerial performance. They concluded that the reduction in agency costs (monitoring costs and inputs/outputs measurement) could improve the sustainability of a firm driven by information technology. However, blockchain is composed of several technologies such as blockchain protocol (consensus mechanism), internet infrastructure, and cloud storage (Davidson et al., 2018). The smart contract faculty of blockchain is a new opportunity to improve the alignments of interests in a decentralized manner (Tapscott and Tapscott, 2016). The transparency and accountability of smart contracts and blockchain hinder opportunistic behavior by either the principal or agent. Every transaction is observable in real-time. There is no successful method to tamper with the common ledger. Although the participant can freely enter the network, all their actions would be encrypted and appended on the chain in a chronological manner. This method of creating a common ledger remarkably improves the traceability of inputs and outputs.

The interreact of two determinants in the agency theory and two blockchain properties addresses the core issue within an organization-moral hazards and conflicts of interest. An immutable data ledger provides traceable trajectories of each input and output, which enables a fair distribution of incentives via a proper-designed incentive protocol. Thus, we propose the following:

Proposition 3a. The monitoring cost of an organization will decrease by adopting blockchain technology to the existing business process.

Proposition $3 \boldsymbol{b}$. The bonding cost of an organization will decrease by adopting blockchain technology to the existing business process.

Proposition 3c. The residual loss of an organization will decrease by adopting blockchain technology to the existing business process.

In addition to the decrease in the agency costs as discussed, blockchain encounters moral hazard risks (honest reporting behavior) when relying upon third parties to verify the registry of assets, as blockchain is heavily dependent on third parties to verify the fidelity of assets registered (Catalini and Gans, 2016). For example, with physical assets like jewelry, an appraiser must be consulted and deferred to in order to verify the value of the asset. Both parties must trust the expertise of the appraiser and grant them the ability to register the "truth" of the asset's value on the register. Here, the appraiser functions as an "oracle."

Since the data on the common ledger triggers the execution of smart contracts, the collection of the data relies on "oracles" to transfer the information from the real-world to the blockchain data registry ${ }^{2}$. The agent's role shifts away from the traditional responsibilities of monitoring business activities in an organization and reporting to the principal, to their new role of supervising those third party "oracles." Since the role of a third-party becomes more significant in this model, a "reputation mechanism" can be another means of regularizing the third parties' opportunistic behavior (Catalini and Gans, 2016). Figure 2 demonstrates the shift in agency costs by adopting blockchain technology. Our fourth proposition is as follows.

Proposition 4. Blockchain shifts the monitoring efforts from internal to external sources and thus increases the search and information costs in transaction cost.

\section{RESEARCH METHODOLOGY}

This study uses a case study as a method and follows Thomas's topology of case study (Thomas, 2011) to analyze a blockchainbased startup company. According to the design frame of case study trajectories-subject, object, purpose, approach and process (Figure 3), we examine the concepts and propositions we laid out in the previous sections in a real-world setting.

\section{Subject}

A key example is selected from a free Initial Coin Offering (ICO) rating platform-ICObench ${ }^{3}$. We use keywords such as "peerto-peer" and" utility token" with expert ratings to filter out the potential cases. The experts rate each company or startup and provide analytical and technical suggestions to the investors. We selected a successful case from the pool, InsurePal, a blockchainbased, decentralized insurance platform that was founded in 2015 and succeeded in raising 18 million dollars through ICO in January 2018 from the backer. The company was honored as a top 10 ICO blockchain solution provider in 2018 and was renamed "Vouchforme" to expand the business scope beyond the insurance industry. In our study, to reduce the complexity of the business process, we focus on the initial scope of InsurePal.

\section{Object}

The analytical focus of our case is the shift in business process due to the introduction of blockchain intervention. We examine our subject through the theoretical lenses of transaction and agency costs. The hypothesized cost transformations are explored and described in detail in the later section.

\section{Purpose}

We are interested in the intrinsic nature of our subject. The exploratory elements such as variables of transaction and agency costs are used to examine our propositions (theory-testing). We aim to investigate a single and retrospective study to thoroughly analyze the business model of InsurPal.

\footnotetext{
${ }^{2}$ http://www.oraclize.it/

${ }^{3}$ https://icobench.com/
} 


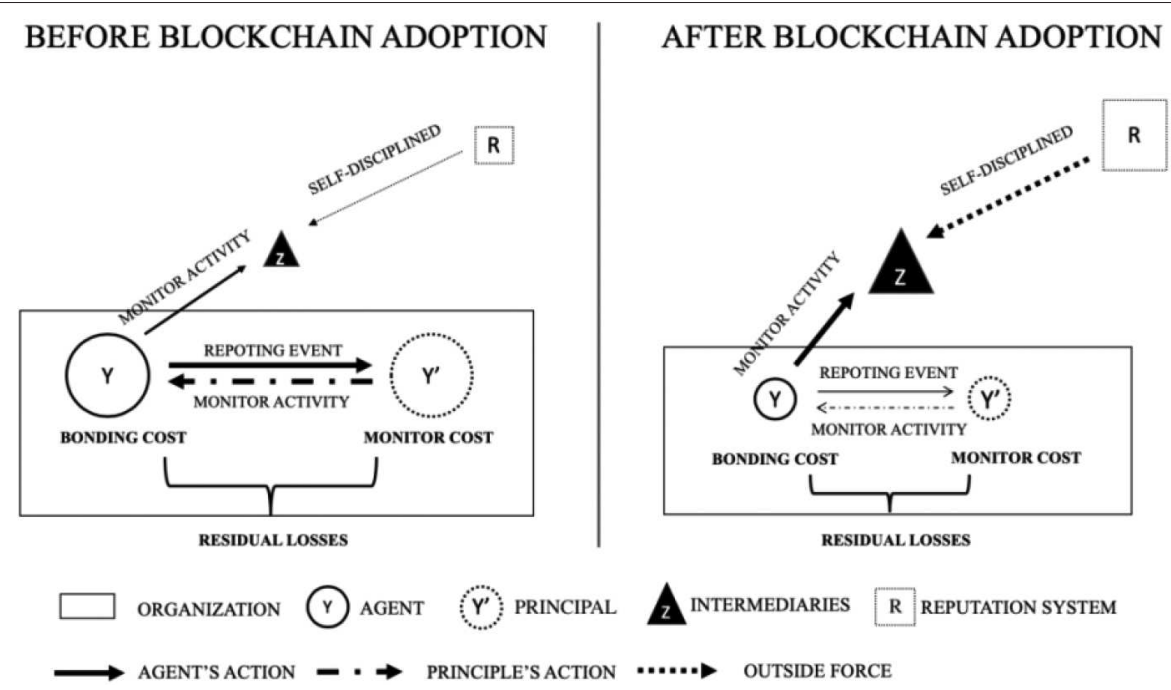

FIGURE 2 | The transformation of agency costs in the adoption of blockchain technology. The size of a node indicates the importance of a role that plays in the network. The role of a third-party $Z$ becomes more crucial and thus the size increases, and so does the importance of reputation mechanism. The thickness of arrows indicates the load of activity. The distance between the agent $Y$ and the principal $Y$ ' implies the consistency of interests from both parties. Adopting blockchain reduces distances between nodes and improves consistency - the shorter the distance, the smaller the residual losses.

FIGURE 3 $\mid$ Case study of cost transformation in a peer-to-peer insurance platform. This diagram is adapted from the design framework of Thomas (2011).

\section{Approach}

To guide our analysis, we adopted a narrative approach involved theoretical elements to develop a detailed business process of our selected insurance commodity. We focus on one of the most common insurance commodities, car insurance, and explore a simple use case of an overpayment issue as the foundation of our case analysis. We collect all the narrative data from the official website ${ }^{4}$, whitepaper ${ }^{5}$, blog posts and news ${ }^{6}$. The official website provides the basic information of platform features and the benefits bring to the insurer and the policyholder. Five news and four blog posts are identified that introduce the related claim process of a car insurance commodity, including

\footnotetext{
${ }^{4} \mathrm{https}: / /$ vouchforme.co/

${ }^{5}$ http://vouchforme.co/VouchForMe_whitepaper_2018.pdf

${ }^{6} \mathrm{https} / / /$ medium.com/vouchforme
}

interviews with the user, press from insurance partners when new insurance products launched, the introduction of how utility tokens work and so forth. The whitepaper gives more detailed knowledge on the business core values, technical overview of social endorsement and blockchain concepts.

\section{Process}

Through examining their whitepaper and official blog, we use variables of transaction and agency costs as key scenarios to collect the information of the corresponding business processes. We mapped out the associated costs from the interviews and description of use cases in the whitepaper and blog posts. For example, while analyzing the search and information costs, we start from a simple use case (i.e., an overpayment problem) illustrated in the whitepaper. In the narrative description, we 
A

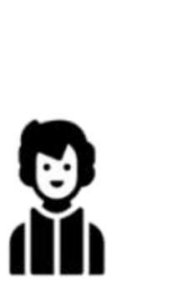

$\$ 1000$ POLICY PAYMRNT
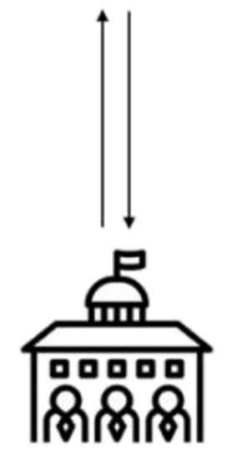

\$1000 PREMIUM FOR CAR INSURANCE
B
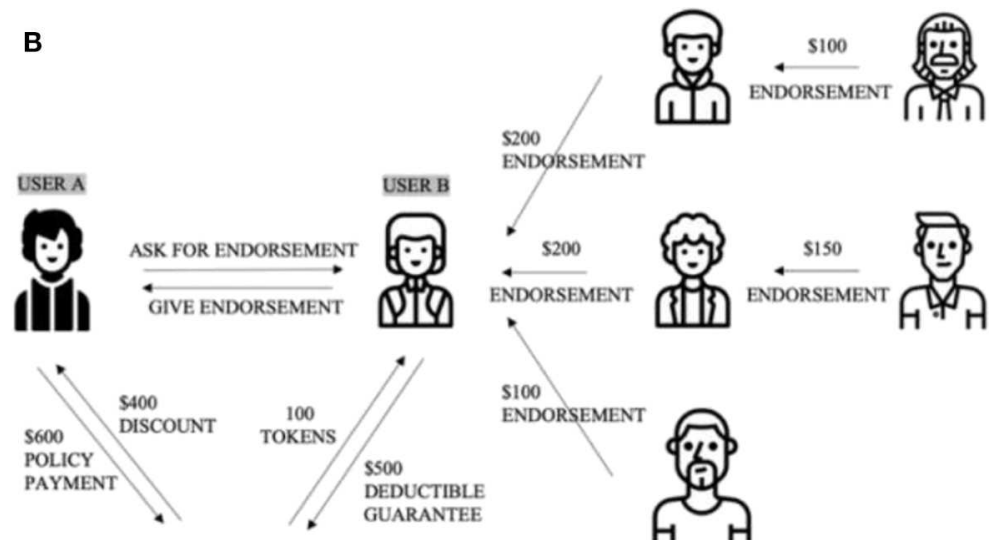

ESTDORSEMENT
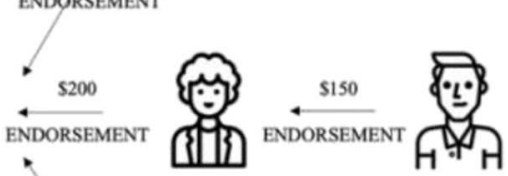

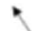

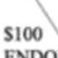

ENDORSEMENT

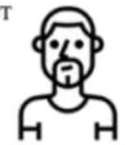

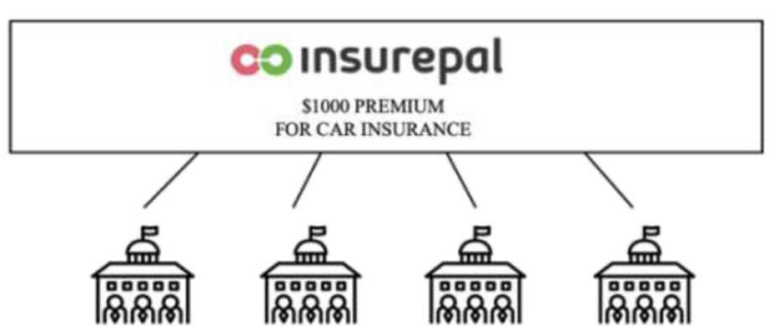

FIGURE 4 | Comparison of business processes of a car insurance premium via (A) a traditional insurance bureaucracy and (B) using InsurePal service.

first define the players (the insurance provider, the policyholder, InsurePal) in the story and the steps involved in a claim process. In each step, we then depict the scenario, explore information flow, and identify the interaction among all stakeholders involved in terms of any information searching behavior (i.e., the collection of personal information when registered to InsurePal).

In our case, an insurance company is the focal organization and uses a third-party platform (InsurePal) to serve their customers. From the perspective of the focal organization, the agent and the principal can be mapped to the manager and the stakeholder of an insurance company. The business process is summarized in the Background information section and the detailed analysis is summarized in sections Transformation of the transaction costs by blockchain intervention, Transformation of the agency costs by blockchain intervention, A two-stage cost transformation in blockchain transition.

\section{Background Information - InsurePal}

InsurePal is a provider of a global, blockchain-based, decentralized insurance platform powered by the social proof mechanism. There are three main players in the ecosystem-the insurer, the policy holder and the blockchain platform provider (InsurePal). Figure 4A shows the traditional relationship between the insurance provider and the policy holder. Figure 4B demonstrates how InsurePal involves and participates in the existing business environment. InsurePal serves as a two-sided platform that connects the two parties together and provides both parties with network benefits. The platform partners with the insurer and uses social endorsements patent to help the policy holder reduce fraud and enable the fair pricing of premiums. On the other side, insurance companies (the agent) take the advantage of the platform to facilitate client segmentation, help risk profiling, reduce fraud and save costs. Since blockchain provides a new way of creating trust among participants by its transparency and traceability, InsurePal not only revolutionizes the process to share risks but also reinforce the reliability of the platform.

Social endorsements could create premium reductions in the following manner (Figure 4): User A would like to avoid overpaying for car insurance. When User A (policyholder) use InsurePal to buy a policy, User A automatically becomes a member of InsurePal and all the data about the policy is shared with InsurePal. User A can seek a social endorsement from User B (the endorser). If User B is willing to endorse, User B also becomes a member of InsurePal and a legal contract is created between them. User B can arbitrarily vouch any amount that User A will be penalized for should a fault claim happen. When User A files a claim, the insurer has to approve and thus User A can use InsurePal to claim the guarantee from the endorser. InsurePal registers User B's financial credentials (i.e., credit card information) in the blockchain ledger as a guarantee for the social endorsement. The amount quarantined as an endorsement to cover part of the deductible returns to User B as utility tokens distributed to their wallet as financial incentives for User B's diligence. The Social Proof TrustScore rates the value of the tokens. In this example, User B could also seek multiple endorsers as co-signers. Each endorser adds a link that furthers its social credit score and allows endorsers to 
distribute risks further through their networks (Figure 4B). Users interact to create a hierarchal social proof network with direct connections between endorsers and indirect links to anonymous risk sharers. Eventually, User A can reduce its insurance premium substantially by its social trust network rather than feeding the bureaucratic apparatus (Figure 4A).

Thus, through the individual's social endorsements, the platform can reduce the policyholders' premium by risk-sharing and enhancing user self-regulation. Compared to traditional endorsements by co-signing, InsurePal provides the policyholder with a new channel to distribute risks by the trustworthy network. The data from the policyholder, the endorser, and the insurer is shared and immutable. Trusts are therefore built relying on the transparent nature of blockchain technology. In addition, InsurePal provides the co-signers (endorsers/nodes on the blockchain) with additional "utility tokens" (digital currency) as incentives to motivate their participation. In this way, everyone can directly or indirectly help anonymous individuals build their credit history in a peer-to-peer manner and be rewarded with a discount on their insurance premium. Eventually, the released tokens become a means for paying for all transactions executed on the platform, achieving an ultimate decentralized system.

\section{Transformation of the Transaction Costs by Blockchain Intervention}

This section answers the research question: How does the introduction of blockchain transform the transaction costs of a firm? and supports our proposition 2 by exploring three types of transaction costs that introduced in section Transaction cost theory. We further discuss the impact of the agency theory on the search and information cost to answer the research question: How do the new transaction costs and agency costs interact and what effects does this have on the organization when adopting blockchain technology to the existing business process? and support our proposition 4.

\section{Search and Information Cost}

The solution to the overpayment problem is analyzed through transaction cost theory to help an insurance provider determine if a policyholder is eligible for a discount. As the description from an official blog post illustrated:

Every year thousands of new drivers pass their tests... With no driving history, insurance is out-of-reach for many new drivers, and even for those who can afford it, which insurance provider what differentiates one insurance company from another?... Insurance companies face a similar dilemma, and each new driver is an opportunity for a new potential customer. Still, without driving history, it's impossible to separate the responsible drivers from the reckless.

Insurance is a service to manage risk. The key information to evaluate a customers' risk level and make an appropriate quote is the data related to a person's behavior. According to the narrative description in the whitepaper:

The insurance costs are determined through classification models that segment people into groups sharing similar data, such as age, sex, marital status, residence, driving record etc. For each of these segments, a "one size fit all" model for all members is calculated, with no or little further differentiation... InsurePal too uses a similar basic algorithm to calculate the client's premium based on a short questionnaire data to be able to undercut the premium price with a social proof by using an advanced InsurePal algorithm. All necessary data can be acquired by a client taking photo of his/her existing policy and in case of motor car insurance, by also taking a photo of his/her driving license and vehicle basic documentation... basic documents are collected to be able to undercut the with social proof algorithm.

The insurance provider's search and information costs include the collection of personal information such as age, address, and marital status, and the evaluation of risk factors such as driving history and health conditions. The information is stored on the blockchain. In terms of the time specificity of a transaction, the distributed and encrypted data ledger shared across the insurance provider, the policyholder, and InsurePal enable immutable and transparent data. Once the policyholder signs a contract with the platform, the information can be synchronized to the common ledger. The time for each party to access data and execute a transaction reduces. According to the whitepaper:

\begin{abstract}
InsurePal is a peer-to-peer insurance product that monetizes the trust between close friends and family through vouching. When someone vouches for a driver, they are confirming they trust them to drive responsibly, and to ensure these endorsements are accurate, vouching will be backed up with a financial guarantee.
\end{abstract}

Furthermore, using the traditional way to evaluate the risks of a policyholder, the insurance provider needs to collect data from each individual and its co-signers. A single framework for risk pooling is inefficient. Blockchain's consensus mechanism and traceability allow a group of people to make common agreements and make the network trustworthy. After the intervention of a blockchain-based social proof system, InsurePal utilizes a unique algorithm to calculate a policyholder's TrustScore, which facilitates the process of risk evaluation by introducing a new dimension-social trust. A group trusted peers support the credit of a policyholder through a peer-to-peer social proof network. The premium is assured by their provided financial assets, enhancing the reliability of a user's TrustScore to finalize risk evaluations. The programmability characteristic of blockchain can further reduce the time for risk pooling (process of search and information cost). According to the whitepaper:

By targeting solely the segment of lower-risk drivers (with a proven history record) the claims frequency significantly reduces, leading the insurances insurers to a better client' segmentation and a significant decrease in policy prices for diligent and responsible clients... The nature of our proposed decentralized business model motivates self-regulation within the community as the risk-prone individuals are automatically expelled from the scheme... As a consequence, even more and larger discounts can be offered to the community instead of feeding the expensive bureaucratic apparatus. 
In addition, the network mechanism of social acquaintances can further segment insurance customers by risk levels so that the risk-seeker (unreliable users) will automatically be filtered out by the community since no one is willing to vouch for a highly unfavorable case. The segmentation can help the insurer position the target clients, providing them with more accurate and proper insurance premiums. The trusted network mitigates the insurance provider's effort to validate a policyholder's risks. As a consequence, the search and information cost will reduce, which supports proposition $2 \mathrm{a}$.

The perspective of agency theory requires a contrary view. The agent confronts the new challenge of how to evaluate the social proof mechanism. This may increase the search and information costs to assess the validity of the algorithm. As indicated in the whitepaper:

The most suitable expression for InsurePal using insurance terminology would be "a third-party deductible." It is proven that a classic deductible reduces insurance claims frequency due to the clients' imposed self-evaluation. From this fact InsurePal proceeded when re-inventing an InsurePal third-party deductible into a third-party evaluation with introducing social proof. By doing so, InsurePal managed to objectivize the evaluation process itself.

Since InsurePal plays a third-party role in the relationship between a policyholder and an insurance provider, the agent does not have control rights over the internal validation of InsurePal's algorithm. Evaluating the performance of a third party becomes a new challenge, and corresponding managerial problems would emerge. For example, the necessity of an organization to increase investment in recruiting blockchain expertise for validity assessment or outsource a blockchain consultant company for help. The alternative efforts made on the evaluation would increase the search and information cost. The maturity of a validation technology/method is crucial in leveraging the changes in the search and information cost. Therefore, proposition 4 is supported when the validation technology is mature. A further discussion on the maturity of validation technology is in section First-stage transformation.

\section{Bargaining Cost}

The traditional process for generating an insurance premium quote requires the policyholder to provide the personal information required for an insurer to assess risk and return a range of premiums. A policyholder can negotiate with the insurer of the premium and find co-signers to argue for a reevaluation. Blockchain intervention through InsurePal requires that the relationship among the participants, such as the policyholder, insurance provider, and endorser, should be defined in detail since the smart contract with automatically execute the agreements by lines of code. An example of use case in the whitepaper illustrated:

Andrew and Susan want to make a business transaction, but don't completely trust each other. If their business arrangement doesn't happen in due time and leads to a more challenging dispute, they begin with an arbitrage. The arbitrage determines who breached the contract and sets the amount of penalties for
InsurePal to cover. They sign a smart contract defining breaching penalty, arbitrage body and arrangement's due date and insure their business transaction with InsurePal.

Each transaction is associated with uncertainty. In the case of InsurePal, the uncertainty of a transaction comes from the trust between the customer and its social acquaintance. Therefore, while building a contract among the policyholder, the endorser and InsurePal, more negotiations are required such as conditions for arbitrage, breaching penalty, etc. Due to the decentralized nature of InsurePal, the human-capital trained to undertake the negotiation tasks also increase. Therefore, the bargaining cost increases in order to reduce transaction uncertainty, which supports the proposition $2 \mathrm{~b}$. In addition, the core value of InsurePal as stated in the whitepaper:

Our vision is to craft an insurance premium based on one's social endorsements by rewarding diligence... The social proof mechanism can be applied to an insured having more than one endorser, too. As each new endorser also has their own social proof network, a hierarchical social proof virtual network establishes. In the event of a claim, one's first (direct) social proof endorsers pay the most, whereas all indirect connections (secondary, tertiary, etc.) pay exponentially less... Blockchain is essentially a permanent and immutable record of transactions within a network... The system that relies on references to other blocks that are cryptographically secure within the digital ledger is much more transparent than traditional approaches to sharing data across a value chain.

Attributed to blockchain's immutability, recovering from adverse effects becomes more costly since more parties are involved. If the rules are not clarified and coded correctly in a smart contract before implementing and installing on each node (participant in blockchain), an "error" in a smart contract is not only impacting a one-to-one relationship between the insurer and the policyholder but also shaking a whole social trust network. Therefore, the resulting bargaining cost will increase to avoid recovering costs. The second increase in the bargaining cost also support our proposition $2 \mathrm{~b}$.

\section{Policing and Enforcement Cost}

Smart contracts are automatically executed within blockchain applications when the "if-then" conditions are met. As indicated in the whitepaper:

The traditional sector is run by large networks with thousands of employees and the vast cost of daily operations and binding contracts preventing them from agility. However, many of their activities are repetitive or redundant without creating value and could easily be replaced by automated data-driven software and further by self-regulating peer-to-peer insurance models... the underwriting of insurance policies, payment system, social proof and even claims handling are dealt with the application of smart contracts, run on the Ethereum public blockchain.

InsurePal uses the Ethereum public blockchain to execute its smart contracts. Those agreements include the underwriting of insurance policies, handling claims and, terms of social proofing. In terms of blockchain's programmability, compared to the 
traditional process of supervising the enforcement of contracts, the required human efforts can be reduced substantially through smart contracts so long as the tasks can be digitized and codable.

In addition to the insurer and the policyholder, InsurePal allows the insured individual's social acquaintance to participate in the network, drastically increasing the size of contract partners. An example of use case retrieved from the blog post:

When Peter opts for a service provider... After registering Peter lands on the policy page where all the data about his policy and options on how much he can save by collecting guarantees. He uses a simple process of inviting his friends and family to vouch for him in the form of a financial guarantee. Peter's pot is filling up with guarantees to a maximum amount of 1000 EUR for the next seven days. When the pot is full or when the deadline of seven days expires, the guarantees become active. At this point, a legal contract is formed between the policyholder and each guarantor and information about the amount of obtained guarantees is sent to the insurance company.

After the introduction of social proof, more contract partners are involved, increasing the uncertainty and complexity of agreements and the difficulties to supervise agreements and parties using the traditional method. The uncertainty of contract enforcement (i.e., human mistakes such as the incorrect input of premiums) becomes lower due to blockchain's programmability. As a consequence, the backbone of blockchain-smart contractwill reduce policing and enforcement costs. The proposition $2 \mathrm{c}$ is hence supported.

\section{Transformation of the Agency Costs by Blockchain Intervention}

This section answers the research question: How does the introduction of blockchain transform the agency costs of a firm? and supports our proposition 3 by exploring three types of agency costs that introduced in section Agency cost theory.

\section{Monitoring Cost}

The principal incurs monitoring costs to supervise the agent's performance to ensure their behavior aligns with the principal's expectations. As the CEO Jacob responded in an interview:

For too long insurance has relied too much on segmentation, which puts people in boxes rather than supporting them based on their individual behavior... Customers want to be recognized as individuals rather than a number, especially younger customers, and insurance companies will have to adapt to changing consumer needs... VFM gives this power to the trusted networks of clients, and uses the digital solutions to ensure that clients pay the premium they deserve for the risks they want to insure... We don't believe statistics should be leading, actual individual behavior should be.

The CEO pointed out the main challenge that the insurance industry encounters. InsurePal consumes the data from the policyholder and all the endorsers to calculate a real-time average value that determines a policyholder's credibility-Social Proof TrustScore. As explained in the whitepaper:
P2P insurance is a risk sharing network where a group of likeminded individuals pool their premiums together to insure against a risk. The membership is selective, meaning the group's peer pressure reinforces responsibility and helps keeping losses down to minimum.

Through a third-party platform, InsurePal, the insurance provider can rely on the TrustScore to mitigate the effort on risk evaluation of each policyholder. The implementation of InsurePal directly contributes to the agent's performance in improving managerial efficiency. The traceability of blockchain makes the accountability of the agent (an insurance company) and a third-party platform (InsurePal) clearer. The principal can always trace an agent's actions by examining records that are registered on-chain. This reduces the effort the principal must exert to control for an agent's potential opportunistic behavior (i.e., monetize from the risk premium). As a consequence, the monitoring costs of the principal will reduce, which supports the proposition 3a. According to the whitepaper:

\begin{abstract}
InsurePal introduces it as a principle of a third party deductible... InsurePal model is self-regulating hybrid of a classical insurance and peer-to-peer endorsements on blockchain, backed with social proof. To avoid any scepticism, InsurePal offers a limited fee guarantee and variety of options to ensure one reasonably saves up compared to traditional policies. Through such endorsements and guarantees, the insurance network in whole becomes more and more risk aware and consequently induces a change of people's behavior to better.
\end{abstract}

Moreover, the role of a third-party (InsurePal) becomes more crucial in the decision-making process. The performance of the back-end social endorsement algorithm determines the reputation of InsurePal, motivating InsurePal toward self-regulation and maintaining a balance in the network. The behavior change of third parties signals the shift in a manager's focus from internal processes toward more external sources. An insurance company (the agent) must have more rigorous control over the quality/validity of third-party (InsurePal) representations and the corresponding technology configurations, increasing the monitoring activities on third parties.

\section{Bonding Cost}

Since blockchain intervention improves transparency, the reduction in information asymmetry can mitigate the agent's opportunistic behavior. The agent is less likely to act against the principals' interests. Sometimes the principal's interests are violated. The unexpected results due to unpredictable system risks should not be attributable to the agent. As indicated in the whitepaper:

The systemic risk is very hard to predict, due to the short time span, and is unique to the industry. Everything from hard forks to new crypto attacks are a source of systemic risk that traditional investments don't suffer from... Many projects are interdependent, which causes dependency risk to projects. For example, a crypto project built on ethereum will be affected by things happening in 
ethereum, like a bug found in a compiler, or an attack on the ethereum network.

Blockchain's traceability plays a role in clarifying the accountability of each participant. For example, if InsurePal suffers a malicious attack, and all the necessary steps are executed in a timely fashion and recorded to the ledger, the principal should not base the failure on the agent. The agent no longer receives the same bonding cost from traditional structures since the traceability of all the actions can prevent the agent from evidence alteration. The principal can clearly identify the accountability of each party, resulting in a reduction in the bonding cost by the agent. Hence, the proposition $3 \mathrm{~b}$ is supported.

\section{Residual Loss}

Loss may incur when the misalignment of interest between the agent and the principal exists. As described above, due to the reduction in both the monitoring cost by the principal and the bonding cost by the agent, the resulting residual loss will further reduce. The derived cost transformation supports the proposition $3 \mathrm{c}$.

\section{A Two-Stage Cost Transformation in Blockchain Transition}

This study aims to answer the question of how the transaction costs and agency costs transform by the introduction of blockchain technology. The ultimate status of a business entity triggered by blockchain is a network with properties of permissionless (peer-to-peer) blockchain applications that is highly autonomous. Every participant can access the network, broadcast new transactions, verify transactions, and append new data blocks on the chain. With the level of decentralization increased and the power of smart contract enhanced, the centralized system will initially grow to the transition stagePermissioned Blockchain with a hybrid structure of both private and permissionless. The overall increase or decrease in the costs diverge case by case. When the aggregated benefits outweigh the overall loss, adopting a blockchain-based system can be worthwhile.

This section answers the research question: What effects does the introduction of blockchain technology have on the boundaries of an organization with respect to IT infrastructure? and supports our proposition 1. InsurePal is an example of a permissioned blockchain service provider that aims to reach the ultimate goal of peer-to-peer insurance (permisionless blockchain). There are two stages of transformation in the evolutionary process. The first stage of transformation is captured by our case analysis and the second stage of transformation is analyzed by reviewing the scenario of InsurePal's ultimate business goals. With respect to research question 4 , we also discuss the interaction effect of the two theories on the search and information cost in section First-stage transformation. The transformation of transaction and agency costs during the evolutionary process is summarized in Figure 5.

\section{First-Stage Transformation Unchanged organization structure}

Moving from a private to permissioned blockchain mitigates the agent-principal problem since the organization structure has not yet transformed; organizations that intend to utilize blockchain technology desire access to its properties like the transparent and immutable ability of recording data to reduce information asymmetry between the agent and principal. In our case study, the focal organization (insurance companies) does not change the organization structure although the business process has been altered by adopting InsurePal. The agent-principal relationship remains the same in the focal organization. As we discussed in section Transformation of the agency costs by blockchain intervention, the transparency and traceability of blockchain mitigate the two main root causes of agency costs-information asymmetry and conflict of interests. The extant study also shows evidence of how blockchain technology reduces agency costs. For example, Lafarre and Van der Elst discuss how blockchain can motivate shareholders' involvement in the annual general meetings (AGMs) (Lafarre and Van der Elst, 2018). AGMs are mandatory events that are used for the principal to determine the performance of the agent and make the most influential decisions on corporate governance. Small shareholders often lack motivation in voting due to the high voting costs over the resulting benefits and tend to defer the monitoring tasks of those who have large shares. The core remote voting system should provide the functions of transparency, verification, and identification (Lafarre and Van der Elst, 2018). Blockchain allows the shareholders to participate in a transparent way of verifying voting decisions. In this transition from a private to a permissioned blockchain, the organization structure remains unchanged, and thus the reduction in agency costs is significant.

\section{Moderator-maturity of validation technology}

Maturity of the validation technology moderates the search and information costs. From the lens of transaction cost theory, the search and information cost will decrease (Proposition 2a) since the process of gathering agreements. Smart contracts can automatically execute the transactions. InsurePal allows the policyholder to reduce unreasonable insurance premiums and helps the insurer (our focal organization) facilitate the process of risk assessment by their patent of social endorsement. When the endorser is willing to vouch for a friend, the binding agreement is automatically generated. As long as the required information collected, the smart contract will execute the agreement and reach the goal of risk sharing. Thus, the search and information cost decrease.

From the agency theory perspective (auditing/monitoring), third parties become more crucial in the blockchain setup. The search and information cost increases (Proposition 4) because additional efforts are required for the agent to validate a third party (i.e., the use of multiple oracles to reduce errors). In our case, InsurePal serves as a third-party service provider, the agent requires additional effort to monitor InsurePal's performance either by hiring professionals or outsourcing. As a consequence, in the first transition stage, the maturity of a third-party 


\begin{tabular}{|c|c|c|c|c|}
\hline & & & & \\
\hline & & & & \\
\hline & & & & \\
\hline & & & & \\
\hline & $\begin{array}{c}\text { PRIVATE } \\
\text { BLOCKCHAIN }\end{array}$ & $\begin{array}{l}\text { PERMISSIONED } \\
\text { BLOCKCHAIN }\end{array}$ & $\begin{array}{l}\text { PERMISSIONLESS } \\
\text { BLOCKCHAIN }\end{array}$ & \\
\hline & $\begin{array}{l}\text { High } \\
\text { transaction } \\
\text { costs }\end{array}$ & $\begin{array}{l}\text { Increase/D } \\
\text { ecrease in } \\
\text { transaction } \\
\text { costs } \\
\text { depends on } \\
\text { scenario }\end{array}$ & $\begin{array}{l}\text { Low } \\
\text { Transaction } \\
\text { costs }\end{array}$ & EXTERNAL ENVIRONMENT \\
\hline & $\begin{array}{l}\text { High } \\
\text { agency } \\
\text { costs }\end{array}$ & $\begin{array}{l}\text { Low } \\
\text { agency } \\
\text { costs }\end{array}$ & $\begin{array}{l}\text { New } \\
\text { format of } \\
\text { agency } \\
\text { costs }\end{array}$ & INTERNAL ORGANIZATION \\
\hline CENTR & ALIZATION & & DECENTR & ALIZATION \\
\hline
\end{tabular}

validation (expertise, oracles, and more) is a key determinant of examining the overall impacts of search and information costs.

When the validation technology is not mature, the impact of agency theory will outperform that of transaction theory on search and information cost. When the validation technology is mature, the impact of transaction theory will outperform that of agency theory on search and information cost.

\section{Second-Stage Transformation \\ Changed organization structure-DAO}

The ultimate goal of InsurePal is to create an environment for a fully decentralized peer-to-peer insurance. Decentralized autonomous organizations (DAO) mature while networks transition from a permissioned to a permissionless blockchain (Figure 3, Column 3). Every participant, the insurer or the policyholder can freely join the network and automatically interact with each other through smart contracts. Although the existing stage of InsurePal still uses credit cards as the main payment method, the ultimate goal is to execute transactions fully by their issued utility tokens. At this stage, the keeper (also known as a validator/miner) joins the network to reinforce the principalagent relationship and alter the role of the agent further. The agent is required to report to the principal and the keeper (Cong and He, 2019). Tokens, a new incentive mechanism, can be embedded in smart contracts to provide the principal with new indicators to measure the agent's performance. The principal evaluates the agent through the current utility value or the discounted expected value of tokens (Cong and He, 2019). The fundamental changes in the principal-agent relationship shift further the boundaries of an organization from hierarchies toward electronic markets (Proposition 1). In addition, Coy and Kharif brought a concept that participants can freely collaborate to form "free agents" to replace the traditional hierarchy business structure ${ }^{7}$. However, there are debates over the idea of the DAO.

From a corporate law perspective, corporations can make more efficient decisions through centralized management. Decision making is inefficient and hence, slower for the DAO, resulting in a sub-optimal situation (Lafarre and Van der Elst, 2018). Although the survival of the DAO is questionable, blockchain facilitates a new format of agency costs in a new organization form and makes improvements in the agent-principal problem through its ability to ensure trust and transparency.

\section{Transaction costs reduction}

Mart contracts facilitate the decrease of transaction costs by providing a new channel for bargaining between autonomous organizations. In a fully peer-to-peer insurance environment, the communication between the insurer and the policyholder can be carried out by smart contracts. The rapid development of artificial intelligence will further lower human effort in bargaining by relegating negotiation tasks between peers partly or wholly to chatbots. In addition, search and information costs for blockchain applications are relatively high. As we discussed in section First-stage transformation, the maturity of validation technology is a key determinant to examine the impact of search and information cost. At the second stage of transformation, when the validation technology becomes mature, search and information costs will be lowered, along with transaction costs.

${ }^{7}$ https://www.bloomberg.com/news/articles/2016-08-25/this-is-your-companyon-blockchain 


\section{DISCUSSION}

\section{Clarification of Blockchain's Firm-Level Impact}

Extant studies forward different understandings on the usage of blockchain, leading to the diverse frameworks available for developing blockchain-based services. The contributions of this study to literature are 3 -fold. First, this study contributes to the existing literature on the understanding of blockchain from an economic prospective. Current studies have been focused on the functionality of a blockchain system from the perspective of general-purpose technology. For example, Ripple views blockchain as a decentralized environment to serve transaction confidentialities and has been devoted to developing the technical framework to avoid centralized control rights $^{8}$. Zheng argues the activities related to blockchain are data recording and immutable databases, which facilitate cloud computing (Zheng et al., 2017). Christidis and Michael believe the blockchain can function as a global "platform" to support the execution of smart contracts (Christidis and Devetsikiotis, 2016). However, Davidson, Primavera, and Jason suggest that the misrepresentation of blockchain as a technology may impede the economic evolution of an organization and call for researches of analyzing blockchain from the economic perspective (Davidson et al., 2018). It is necessary to analyze how the technology impacts the overall organization to facilitate the strategic alignment between corporation governance and new technology intervention. Therefore, building upon study (Davidson et al., 2018), we use transaction cost theory and further incorporate the agency theory as a foundation to examine the transformation/interaction of costs during different stages of blockchain evolution.

Second, we observe the changes in organizational and managerial structures in different stages of blockchain transition. Malone et al. (1987) predicted the shift in organizational boundaries while internet technology emerged. We contribute to the work by arguing that Internet-based blockchain technology facilitates the transition of the existing organization toward a more decentralized structure.

Finally, our findings contribute to transaction cost theory (Williamson, 1985). Firms are built to govern the opportunistic behavior of a transaction. We find the potential determinants that would impact transaction costs. The role of third parties becomes crucial in the validating cost of a transaction. The effect of the search and information cost is contingent on the maturity of validation technology. A deeper understanding of how blockchain transforms transaction and agency costs from an institutional viewpoint will benefit strategy development and corporate governance.

\section{Implications on the Blockchain Business Model}

This study implies the potential to facilitate the development of blockchain business models and contributes to the practice.

\footnotetext{
${ }^{8}$ https://z.cash/wp-content/uploads/static-og/static/

R3_Confidentiality_and_Privacy_Report.pdf
}

Applying a single managerial methodology to different Blockchain configurations is risky. A deeper exploration of the strategies should be conducted to avoid failure in using inappropriate business models rather than unquestioningly embracing new technology. For example, we use InsurePal as an example to explore our discussed cost transformation. The insurer adapts InsurePal to enhance business value, change the cost structure and reshape revenue streams. Especially, how an organization interacts with new business partners or whether a new channel change the existing customer relationships determines the shift in an existing business model. However, only a few studies (Nowiski, 2017; Morkunas et al., 2019) discussing the impact of blockchain on business models. Hence, by identifying the fundamental shift in cost structures and organization forms, as we discussed in section Research Methodology, the manager is able to make a proper configuration of corporate resources to innovate a business model.

\section{CONCLUSION AND LIMITATIONS}

Our study proposes that blockchain technology brings two more benefits, trust and transparency, to the existing Internet-based business services, and helps improve corporate governance. Smart contracts improve the execution time of transactions significantly and increase transaction volume rapidly. As the internet shifts hierarchies toward electronic markets, lack of trust between peers inhibits exchanges. Blockchain applications provide a framework for building trust between peers through its consent mechanism, which allows organizations to construct trust and operate in a more decentralized manner. Thus, by including blockchain in the current Internet infrastructure, the decision boundary of organization forms would extend outward.

To answer our research questions 2 and 3, we argue the transformation of costs in different stages of the blockchain transition has different managerial implications for the organization structure and the role of third parties. While transit from a private to a permissioned blockchain, the search and information costs and the policy and enforcement costs will reduce but the bargaining costs will increase. The overall agency costs reduce since organization structures remain unchanged. Third parties play an important role in reporting the truth to the common ledger and leverage the impact of transaction costs on organizations. Thus, the interaction effect of transaction and agency theories on the search and information cost as discussed in section Transformation of the transaction costs by blockchain intervention and First-stage transformation is contingent on the maturity of validation technology, which response to our research question 4 .

However, the transition from a permissioned to a permissionless blockchain implies changes in organizations. A new player (the Keeper) participates in the traditional principalagent relationship. New mechanisms to evaluate a firm's performance such as the current value of tokens emerge. With respect to research question 1 on the organization boundaries, the essential shifts in the principal-agent relationships push the organization structures from hierarchies toward more electronic 
markets as discussed in section Second-stage transformation. The transaction costs are expected to be reduced eventually due to the growing power of validation technology and smart contract. Blockchain does not assume away transaction and agency costs but pushes the transformation of the two, forming a more efficient economic entity.

There are several limitations to this study that should be considered. One of the potential limitations is the selection of blockchain use cases. Due to the blockchain hype, countless of blockchain applications are available to select. However, different understandings of blockchain result in diverse utilization of blockchain technology such as data management, identity management or immutable database. We consider a use case that has all the blockchain characteristics-immutability, transparency, programmability, and decentralization. There are no measurements to technically evaluate the rationality of a use case. Second, our motivation stems from the diversity of blockchain architecture across organizations. Although we propose a new perspective to

\section{REFERENCES}

Abadi, J., and Brunnermeier, M. (2018). Blockchain economics (No. w25407). National Bureau of Economic Research.

Agarwal, R., and Lucas, H. C. (2005). The information systems identity crisis: focusing on high-visibility and high-impact research. MIS Quart. 29, 125-129. doi: $10.2307 / 25148689$

Alchian, A. A., and Demsetz, H. (1972). Production, information costs, and economic organization. Am. Econ. Rev. 62, 777-795.

Ang, J. S., Cole, R. A., and Lin, J. W. (2000). Agency costs and ownership structure. J. Finance 55, 81-106. doi: 10.1111/0022-1082.00201

Bahga, A., and Madisetti, V. K. (2016). Blockchain platform for industrial internet of things. J. Softw. Eng. Appl. 9, 533-546. doi: 10.4236/jsea.2016.910036

Baliga, A. (2017). Understanding Blockchain Consensus Models. Persistent.

Cachin, C., and Vukolić, M. (2017). Blockchain consensus protocols in the wild. arXiv [Preprint]. arXiv:1707.01873.

Catalini, C., and Gans, J. S. (2016). Some Simple Economics of the Blockchain. National Bureau of Economic Research No. w22952.

Chedrawi, C., and Howayeck, P. (2018). Audit in the Blockchain era Within a Principal-Agent Approach. Information and Communication Technologies in Organizations and Society (ICTO 2018): Information and Communications Technologies for an Inclusive World.

Chircu, A. M., and Kauffman, R. J. (1999). Strategies for Internet middlemen in the intermediation/disintermediation/reintermediation cycle. Electronic Markets 9 , 109-117. doi: 10.1080/101967899359337

Christidis, K., and Devetsikiotis, M. (2016). Blockchains and smart contracts for the internet of things. IEEE Access 4, 2292-2303. doi: 10.1109/ACCESS.2016.2566339

Cong, L. W., and He, Z. (2019). Blockchain disruption and smart contracts. Rev. Financial Stud. 32, 1754-1797. doi: 10.1093/rfs/hhz007

Davidson, S., De Filippi, P., and Potts, J. (2018). Blockchains and the economic institutions of capitalism. J. Instit. Econ. 14, 639-658. doi: $10.1017 /$ S1744137417000200

Foss, N. J. (2003). Selective intervention and internal hybrids: Interpreting and learning from the rise and decline of the Oticon spaghetti organization. Organiz. Sci. 14, 331-349. doi: 10.1287/orsc.14.3.331.15166

Holmstrom, B. (1979). Moral hazard and observability. Bell J. Econ. 10, 74-91. doi: $10.2307 / 3003320$

Jensen, M. C., and Meckling, W. H. (1976). Theory of the firm: managerial behavior, agency costs and ownership structure. J. Finan. Econ. 3, 305-360. doi: 10.1016/0304-405X(76)90026-X examine blockchain technology from a firm-level lens, we do not provide a framework to suggest how should a firm deploys blockchain. Also, we only consider a single use case, which limits the possibility of different scenarios that might conflict with our findings and restrict the generalizability of this study.

\section{AUTHOR CONTRIBUTIONS}

R-TS, H-LC, and MS developed the research idea, research propositions, and initiate the case study. AG and WH then joined the research team and provide new perspectives to refine the case study methodology and contributed to the research findings.

\section{FUNDING}

This research is supported partly by State Farm Foundation and Gies College of Business.

Kaal, W. A. (2019). "Blockchain solutions for agency problems in corporate governance," in Economic Information to Facilitate Decision Making, eds K. R. Balachandran (World Scientific Publishers). U of St. Thomas (Minnesota) Legal Studies Research Paper No. 19-05. doi: 10.2139/ssrn.3373393

Kettinger, W. J., Grover, V., Segars, A. H., and Guha, S. (1994). Strategic information systems revisited: a study in sustainability and performance. MIS Quart. 18, 31-58. doi: 10.2307/249609

Khan, H. (2011). A literature Review of Corporate Governance. IPEDR 1:25.

Lafarre, A., and Van der Elst, C. (2018). Blockchain Technology for Corporate Governance and Shareholder Activism. Law Working Paper No. 390/2018; Tilburg Law School Research Paper No. 2018-7. European Corporate Governance Institute (ECGI). doi: 10.2139/ssrn.31 35209

Lajili, K., and Mahoney, J. T. (2006). Revisiting agency and transaction costs theory predictions on vertical financial ownership and contracting: electronic integration as an organizational form choice. Manag. Decision Econ. 27, 573-586. doi: 10.1002/mde. 1275

Levinthal, D. (1988). A survey of agency models of organizations. J. Econ. Behav. Organiz. 9, 153-185. doi: 10.1016/0167-2681(88)9 0071-6

Mahoney, J. T. (1992). The choice of organizational form: vertical financial ownership versus other methods of vertical integration. Strategic Manag. J. 13, 559-584. doi: 10.1002/smj.4250130802

Mahoney, J. T. (2004). Economic Foundations of Strategy. Thousand Oaks, CA: Sage Publications.

Malone, T. W., Joanne, A., and Robert, B. I., (1987). Electronic markets and electronic hierarchies. Commun. ACM 30, 484-497. doi: 10.1145/214762.214766

Morkunas, V. J., Paschen, J., and Boon, E. (2019). How blockchain technologies impact your business model. Business Horizons 62, 295-306. doi: 10.1016/j.bushor.2019.01.009

Nowiski, W, and Kozma, M. (2017). How can blockchain technology disrupt the existing business models? Entrepr. Business Econ. Rev. 5, 173-188. doi: 10.15678/EBER.2017.050309

Ouchi, W. G. (1979). A conceptual framework for the design of organizational control mechanisms. Manage. Sci. 25, 833-848. doi: 10.1007/978-1-4899-7138-8_4

Peters, G. W., and Panayi, E. (2016). "Understanding modern banking ledgers through blockchain technologies: future of transaction processing and smart contracts on the internet of money," in Banking Beyond Banks and Money (Cham: Springer), 239-278. 
Ritchie, B., and Brindley, C. (2000). Disintermediation, disintegration and risk in the SME global supply chain. Manage. Decision 38, 575-583. doi: 10.1108/00251740010378309

Swan, M. (2015). Blockchain: Blueprint for a New Economy. O'Reilly Media, Inc.

Tapscott, D., and Tapscott, A. (2016). Blockchain Revolution: How the Technology Behind Bitcoin Is Changing Money, Business, and the World. London: Portfolio.

Thomas, G. (2011). A typology for the case study in social science following a review of definition, discourse, and structure. Qual. Inquiry 17, 511-521. doi: $10.1177 / 1077800411409884$

Williamson, O. (1985). The Economic Institutions of Capitalism. New York, NY: Free Press.

Williamson, O. E. (1975). Markets and Hierarchies: Analysis and Antitrust Implications. New York, NY: Macmillan Publishers.

Williamson, O. E. (1983). Credible commitments: Using hostages to support exchange. Am Econ Rev. 73, 519-540.

Yli-Huumo, J., Ko, D., Choi, S., Park, S., and Smolander, K. (2016). Where is current research on blockchain technology?-a systematic review. PLoS ONE 11:e0163477. doi: 10.1371/journal.pone.01 63477
Zenger, T. R. (1994). Explaining organizational diseconomies of scale in RandD: agency problems and the allocation of engineering talent, ideas, and effort by firm size. Manage. Sci. 40, 708-729. doi: 10.1287/mnsc. 40.6.708

Zheng, Z., Xie, S., Dai, H., Chen, X., and Wang, H. (2017). “An overview of blockchain technology: architecture, consensus, and future trends," in 2017 IEEE International Congress on Big Data (BigData Congress) (IEEE), 557-564.

Conflict of Interest: The authors declare that the research was conducted in the absence of any commercial or financial relationships that could be construed as a potential conflict of interest.

Copyright (c) 2020 Sun, Garimella, Han, Chang and Shaw. This is an open-access article distributed under the terms of the Creative Commons Attribution License (CC $B Y)$. The use, distribution or reproduction in other forums is permitted, provided the original author(s) and the copyright owner(s) are credited and that the original publication in this journal is cited, in accordance with accepted academic practice. No use, distribution or reproduction is permitted which does not comply with these terms. 